\title{
Correspondence
}

Epidemiol. Infect. (2016).

doi:10.1017/S0950268815001077

first published online 26 June 2015

\section{Erythema multiforme after orf virus infection}

To the Editor:

Joseph and collaborators [1] described two complex cases of orf-associated erythema multiforme (EM) and reviewed the literature, highlighting important clinical, epidemiological and immunological aspects of this condition. One of their cases was a man who developed the disease after religious slaughtering. A similar case was recently observed by our research group. A 34-year-old man presented with a cutaneous papular rash on the trunk, arms and extremities. These itchy papules were erythematous, ranging in size from a few millimetres to $2 \mathrm{~cm}$. Some of the papules joined together, taking on rosette-like shapes (Fig. 1a, $b$ ). There was no fever, lymphadenopathy or other general symptoms. Two days previously, a physician had prescribed an antibiotic because of infection in a wound on the left thumb, which had occurred while slicing raw lamb 15 days earlier. Histological examination revealed a hyperkeratotic and spongiotic epidermis and marked oedema of the superficial dermis with lymphocytic infiltrate and intraepidermal exocytosis (Fig. 1c, d), leading us to suspect EM. Serological analyses, throat swab, and chest X-ray allowed us to exclude hepatitis B virus, hepatitis $\mathrm{C}$ virus, cytomegalovirus, Epstein-Barr virus, herpes simplex virus (HSV), varicella-zoster virus and Mycoplasma pneumoniae infections. Since clinical observation also revealed a brownish and painless erythematous nodule with a central necrotic area on the left thumb (Fig. 1a), a concomitant orf virus (OV) infection was suspected.
To confirm our clinical suspicion, a fresh specimen collected from the thumb wound and a paraffin-embedded skin sample from an erythematous lesion were analysed in order to identify the presence of parapoxvirus DNA. The purified DNA was amplified by Sybr Green real-time PCR and confirmed a parapoxvirus infection [2]. Subsequent sequencing of a B2L fragment amplified by PCR, performed on the DNA extracted from the scab sample and the EM lesion, confirmed an OV infection complicated by EM. After 4 days of treatment with a minimal dose of systemic corticosteroids the EM lesions started to improve. Rifamycin was applied topically and oral tetracycline given for 6 days. Complete regression was achieved 1 month after onset.

To our knowledge, this is the first time that OV DNA has been identified in paraffin-embedded specimens taken from disseminated erythematous lesions. This finding, together with the absence of the typical OV histopathological features in the EM specimens, led us to speculate that the pathogenetic mechanism of $\mathrm{OV}$-associated EM might be similar to that described for HSV, i.e. the result of a cell-mediated immune reaction against viral antigen positive cells [3]. For this reason, we propose that $\mathrm{OV}$-induced EM might be the consequence of a viral dissemination in the bloodstream that can be facilitated by knife wounds. It is intriguing to note that a number of $\mathrm{OV}$-associated EM cases have been documented as a complication of infections contracted after religious slaughtering [4]. As has been shown for HSV the virus present in blood can be phagocytosed by circulating blood mononuclear cells, such as macrophages and Langerhans cell progenitors, allowing the viral fragmented DNA to be transported to the epidermis and transferred to the keratinocytes. The increased regulation of transmembrane protein expression might increase the binding of virus-containing 


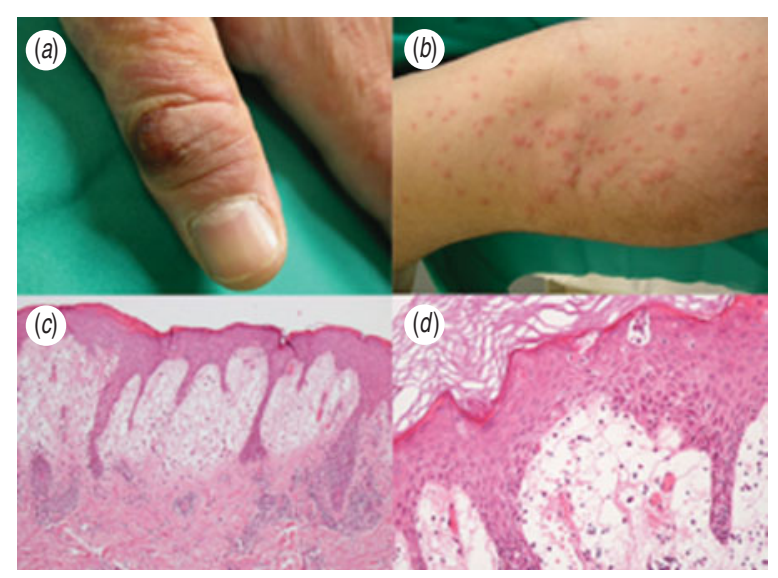

Fig. 1. (a) Infected cutaneous nodule at the first finger of the left hand, cutaneous papulo-vesicular rash on the left arm. (b) Itchy papules were erythematous, ranging in size from a few millimetres to $1-2 \mathrm{~cm}$. Some of the papules joined together, taking on rosette-like shapes. (c) Skin biopsy showing hyperkeratosis and spongiosis of the epidermidis, oedema of the papillary dermis and dermic lymphoid infiltrate with epidermal esocytosis. Haematoxylin and eosin (original magnification 10×). (d) Haematoxylin and eosin (original magnification $20 \times$ ).

Langerhans cells to endothelial cells, accounting for the dermal inflammatory response. A number of OV infections, complicated by EM, have reported in Muslim people after religious slaughtering, so it could be interesting also to investigate if, apart from the peculiar way of infection that might facilitate a temporary blood dissemination of the virus, there can be any other predisposing factors related to ethnic background [5, 6]. A significantly higher prevalence of HSV-associated EM was in fact found in patients carrying particular HLA alleles; for this reason it might be reasonable to investigate the population-specific contribution of the haplotype leading to this complication.

In conclusion, $\mathrm{OV}$ needs to be included as a further cause of EM, and additional investigations of clinical cases might contribute to our understanding of the pathogenesis of $\mathrm{OV}$ infections in humans. The number of reports on complicated $\mathrm{OV}$ infections after religious slaughtering, seem to suggest that predisposing factors related to ethnic background may contribute to the condition.

\section{DECLARATION OF INTEREST}

None.

\section{REFERENCES}

1. Joseph RH, et al. Erythema multiforme after orf virus infection: a report of two cases and literature review. Epidemiology and Infection 2015, 143: 385-390.

2. Gallina $\mathbf{L}$, et al. A real time PCR assay for the detection and quantification of orf virus. Journal of Virological Methods 2006, 134: 140-145.

3. Sokumbi O, Wetter DA. Clinical features, diagnosis, and treatment of erythema multiforme: a review for the practicing dermatologist. International Journal of Dermatology 2012, 51: 889-902.

4. Nougairede A, et al. Sheep-to-human transmission of Orf virus during Eid al-Adha religious practices, France. Emerging Infectious Diseases 2013, 19: 102-105.

5. Khalil I, et al. HLA DQB $1 * 0301$ allele is involved in the susceptibility to erythema multiforme. Journal of Investigative Dermatology 1991; 97: 697-700.

6. Benedek G, et al. Genetics group. Opposing effects of the HLA-DRB1*0301-DQB1*0201 haplotype on the risk for multiple sclerosis in diverse Arab populations in Israel. Genes and Immunity 2010, 11: 423-431.

L. GALLINA ${ }^{1}$, F. VERONESE ${ }^{2}$, P. FARINELLI ${ }^{2}$,

R. BOLDORINI ${ }^{3}$, G. DELROSSO ${ }^{2}$, E. COLOMBO ${ }^{2}$,

E. MALDi ${ }^{3}$, A. PELI $^{1}$, A. SCAGLIARINI ${ }^{1, *}$

${ }^{1}$ Dipartimento di Scienze Mediche Veterinarie, Alma Mater Studiorum Università di Bologna, Ozzano Emilia (Bologna), Italy

${ }^{2}$ Clinica Dermatologica, Maggiore della Carità Hospital,

Università del Piemonte Orientale, Novara, Italy

${ }^{3}$ Unità di Anatomia Patologia, Maggiore della Carità

Hospital, Università del Piemonte Orientale, Novara, Italy

*Author for correspondence:

Professor A. Scagliarini, Dipartimento di Scienze Mediche Veterinarie, Alma Mater Studiorum Università di Bologna, Via Tolara di sopra 50, 40064 Ozzano Emilia (Bologna),

Italia

(Email: alessand.scagliarini@unibo.it) 